\title{
Canada calls in US nuclear power experts to boost performance
}

[QUEBEC] In what its president has called “a humbling experience", the Canadian public utility that was formerly considered a world leader in nuclear power has had to hire US experts to improve the performance of its nuclear power plants.

Carl Andognini, who has 30 years' experience with major US utilities, is to take over as head of Ontario Hydro's nuclear division by the end of this month, assisted by six other US nuclear engineers. A spokesman for the company said it felt that the kind of expertise it needed did not exist in Canada.

The US team was hired on the orders of Allan Kupcis, president of Ontario Hydro. "We've got into believing our own headlines that we're at the top of the world," says Kupcis, referring to boasts during the 1980s that the utility's Candu heavy-water reactors were achieving maximum output for more than 80 per cent of the time. The average has dropped to just under 70 per cent.

Nuclear power accounts for at least 60 per cent of Ontario Hydro's energy output. But over the past ten years the efficiency of its plants has steadily diminished. The Candu reactors are designed and sold by Atomic Energy of Canada Ltd (AECL).
Officials at the design company and the utility say that the problems are not the fault of the technology, but arise from a lack of management expertise that has led to sloppiness in maintenance. For example, a piece of wood was once left in a reactor during repairs. By the time it was retrieved it had disintegrated, causing a disruption to power supplies. On another occasion a lead blanket was left behind by workmen.

A spokesman for the Atomic Energy Control Board, the nuclear-power regulating agency, says the board has been "badgering" Ontario Hydro for some time to improve its safety practices. The Pickering plant, for example, has been given only a six-month licence to operate.

Elsewhere in Canada, there have been mechanical problems with reactors. Reactors at Point Lepreau in southern New Brunswick and Gentilly in Quebec have experienced serious corrosion in the feeder pipes that carry heavy water from fuel channels to boilers to produce steam.

The corrosion seems to be caused by a combination of temperature, mass velocity and iron concentration in the water. These reactors use boiling water. Although there has been some corrosion in the Ontario reactors, which operate at a lower temperature, the problem was not expected to become serious for 20 years.

AECL engineers believe they know how to reduce the corrosion. But they are awaiting results of experiments at Chalk River laboratories, in Ontario, for a definitive answer. Meanwhile, they will provide directives for interim changes by the end of this year.

Candu reactors have been sold to Argentina, South Korea, China and Romania. Argentina's suffers from corrosion, but less than at Point Lepreau and Gentilly, for reasons that are not completely understood. South Korea's reactor is experiencing a more serious problem. Romania's reactor has only just started operation, and has not so far developed any problems.

AECL engineers hope that the corrosion problem will not affect foreign sales. "We've got solutions and we can change materials," says one. "In reactors that we have, we've changed the composition of the steels, so we can avoid the problem in that way." He says that any change to operating conditions will be applied abroad.

DavidSpurgeon

Japan fishes for more space launches

[TOKYO] Japan plans to renegotiate an agreement with fishermen in the vicinity of its two space-launch centres in southern Japan - almost doubling its annual payments to fishing cooperatives of $¥ 400$ million (US\$3.5 million) - to allow launches on a greater number of days each year.

The move is part of a bid to increase the number of launches for its two space agencies for both commercial and scientific missions. There will also be an expansion of launch facilities for the National Space Development Agency (NASDA) which will be used for both commercial and government-sponsored launches.

The Science and Technology Agency (STA) will soon start negotiations with cooperatives in the prefectures of Kagoshima, Miyazaki and Oita in the southern island of Kyushu, and Ehime and Kochi in the neighbouring island of Shikoku. These are relatively close to the launch centre of the Institute of Space and Astronautical Science (ISAS) on the coast of Kagoshima and that of NASDA, just off the Kagoshima coast on Tanegashima island.

Fishermen claim that launches could damage fishing grounds, and the present agreement with them limits each space agency to making launches during two periods of 45 days - one in winter and one in summer. This effectively restricts each agency to a maximum of two launches a year.

The payment to the fishing cooperatives is made by STA as part of the agreement to allow the 90-day launch period. The science agency has budgeted $¥ 700$ million — almost twice the current amount - for the fishermen in its fiscal 1997 budget, which begins on 1 April, to expand the period.

Both the STA, which funds NASDA, and the Ministry of Education, Science, Sports and Culture (Monbusho), which oversees ISAS, want a longer period. NASDA has just begun building a new $¥ 25$-billion (US\$217million) launch pad on Tanegashima, due for completion early in 2000. Along with the existing pad, this will allow launches in rapid succession.

Completion of the new pad is timed to coincide with the first launch of a more economical version of NASDA's $\mathrm{H}-2$ rocket, the H-2A (see Nature 378, 527; 1995). The H-2A will be used by both NASDA and Rocket Systems Corporation, a private consortium of 73 Japanese aerospace companies, banks and insurance companies which was set up in 1990 to sell launches of the H-2 (see Nature 345, 191; 1990).

The corporation has agreed with Hughes Space and Communications International of the United States to make 20 launches, in a five-year period from 2000, carrying commercial satellites.

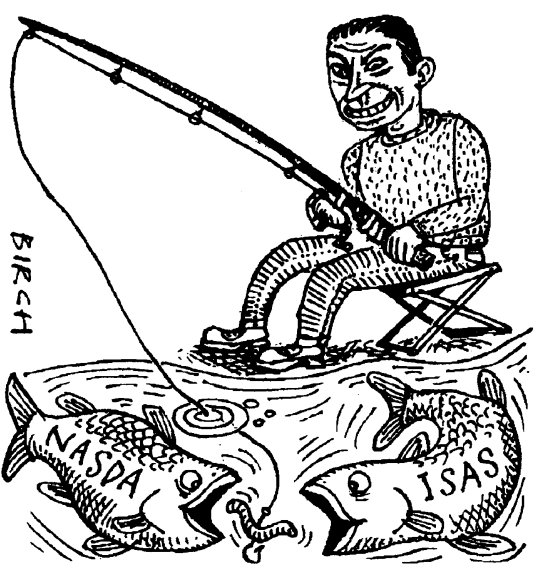

NASDA also expects to increase its number of launches to launch and service the Japanese module for the international space station which is due to start operations early next century. And both NASDA and ISAS have ambitions to carry out scientific interplanetary missions that will require greater flexibility in launch periods (see Nature 370, 405: 1994).

STA is not prepared to say what launch period it hopes to agree with the fishermen, as that is "a matter for negotiation". An agency official stresses that the $¥ 700$ million will not be paid in cash, and it is not "compensation". Rather, it is intended to assist fishermen by providing new facilities such as improved refrigerators.

DavidSwinbanks 